\title{
Effects of Low vs. High Fermionic Modes on Hadron Mass Generation
}

\section{Denissenya*}

Institut für Physik, FB Theoretische Physik, Universität Graz

E-mail: mikhail.denissenvaduni-graz.at

\section{Ya. Glozman}

Institut für Physik, FB Theoretische Physik, Universität Graz

E-mail: Leonid.qlozmanduni-graz.at

\section{B. Lang}

Institut für Physik, FB Theoretische Physik, Universität Graz E-mail: Christian.langduni-graz.at

\section{Schröck}

Institut für Physik, FB Theoretische Physik, Universität Graz

E-mail: mario.schroeck@uni-graz.at

\begin{abstract}
A nonvanishing spectral density of the low-lying eigenmodes of the Dirac operator naturally is a signal for dynamical chiral symmetry breaking $(\mathrm{D} \chi \mathrm{SB})$ via the Banks-Casher relation. The low-lying eigenmodes alone saturate the pseudoscalar channel and the corresponding propagator successfully reproduces the pion mass. In this paper we investigate the effects on the mass generation of hadrons other than pions. The evolution of these masses upon inclusion of an increasing number of the low-lying eigenmodes is confronted with the hadron mass spectrum upon removal of such eigenmodes.
\end{abstract}

31st International Symposium on Lattice Field Theory LATTICE 2013

July 29 - August 3, 2013

Mainz, Germany

\footnotetext{
* Speaker.
} 


\section{Introduction}

In this contribution we study the question to what extent the quark condensate of the vacuum (or, better to say, the physics that is responsible for the dynamical chiral symmetry breaking) contributes also to the hadron mass generation. Such a question was already addressed on a qualitative level in lattice studies [ [ [] ]. One of the conclusions was that the pion mass is saturated by the physics related to spontaneous breaking of chiral symmetry, in agreement with general theoretical expectations. We ask, however, whether it is also so or not for some other low-lying states, like $\rho$-meson, nucleon and $a_{1}$-meson. According to many different model views their mass generation is also related to the chiral symmetry breaking dynamics. This study is complementary to our previous work [B], 团], where we investigated what happens with these hadrons if we artificially restore chiral symmetry by removing of the low-lying modes from the quark propagators.

The density of the low-lying Dirac eigenmodes is related to the order parameter of $\mathrm{D} \chi \mathrm{SB}$, namely, the quark condensate [ [] $]$. The number of these eigenmodes responsible for the quark condensate scales with the lattice volume. We construct hadron propagators from quark propagators considering only a subset of eigenmodes of the spectral representation of the Dirac operator. The propagator built from $k$ low-lying modes only, we call a truncated propagator. A propagator constructed by removing the low-lying modes from the complete set, we call a reduced propagator. The truncated propagator is given by

$$
S_{L M(k)}=\sum_{i=1}^{\mathbf{k}} \frac{1}{\lambda_{i}}\left|\lambda_{i}\right\rangle\left\langle\lambda_{i}\right|
$$

where $\lambda_{i}$ and $\left|\lambda_{i}\right\rangle$ are the eigenvalues and eigenmodes of the chirally improved Dirac Operator $\gamma_{5} D_{C I}[$ [G]. By constructing the reduced quark propagator,

$$
S_{R D(k)}=S_{F u l l}-S_{L M(k)},
$$

we get access to the complementary part of the (high-lying) eigenmodes. We performed our studies on 160 gauge configurations with $n_{f}=2$ dynamical fermions with a pion mass $M_{\pi}=322(5)$ $\mathrm{MeV}$. The lattice size and the lattice spacing are $16^{3} \times 32$ and $a=0.144(1) \mathrm{fm}$, respectively. We choose values of $k=32,64$, and 128 where approximately 32 eigenmodes correspond to $30 \mathrm{MeV}$ [B]. Furthermore the extended set of interpolators enables us to use the variational method. By constructing the cross-correlation matrix

$$
C_{i j}(t)=\left\langle 0\left|\mathscr{O}_{i}(t) \mathscr{O}_{j}^{\dagger}(0)\right| 0\right\rangle,
$$

and solving generalized eigenvalue problem

$$
C(t) \vec{v}_{n}=\tilde{\lambda}^{(n)}(t) C\left(t_{0}\right) \vec{v}_{n},
$$

we obtain the masses of the ground and possibly excited states from the exponential behaviour of the eigenvalues $\tilde{\lambda}^{(n)}\left(t, t_{0}\right)=\mathrm{e}^{-E_{n}\left(t-t_{0}\right)}\left(1+\mathscr{O}\left(\mathrm{e}^{-\Delta E_{n}\left(t-t_{0}\right)}\right)\right)$. These are extracted for each truncation level $k$ in a given quantum channel. The quantum channels and corresponding interpolators are listed in Tab. 四.

Notations $n, w$ and $\partial_{i}$ stand for the Jacobi smeared -narrow, -wide and derivative sources respectively. 


\begin{tabular}{|c|c|c|c|c|c|}
\hline \hline$\#$ & $\rho$ & $\#$ & $a_{1}$ & $\#$ & $N^{+}$ \\
\hline 8 & $\bar{q}_{w} \gamma_{k} \gamma_{t} q_{w}$ & 1 & $\bar{q}_{n} \gamma_{k} \gamma_{5} q_{n}$ & 1 & $\varepsilon_{a b c} q_{a}\left(q_{b}^{T} C \gamma_{5} q_{c}\right), n(n n)$ \\
17 & $\bar{q}_{\partial_{i}} \gamma_{k} q_{\partial_{i}}$ & 4 & $\bar{q}_{w} \gamma_{k} \gamma_{5} q_{w}$ & 18 & $\varepsilon_{a b c} i q_{a}\left(q_{b}^{T} C \gamma_{t} \gamma_{5} q_{c}\right), w(w w)$ \\
\hline
\end{tabular}

Table 1: Number of interpolators (\#) and the interpolators for the $\rho\left(1^{--}\right), a_{1}\left(1^{++}\right)$mesons and the $N^{+}$ nucleon.

\section{Hadron masses with and without the low modes}

We confirmed that the pseudoscalar channel is saturated by the low modes only [四]. Here we consider $k=128$ as a sufficient number of the low modes to reproduce the mass of a pion within error bars. On the other hand when one removes the same number of the low modes from the complete set one artificially removes the pion from the spectrum. We here determine quantitatively the effects of $\mathrm{D} \chi \mathrm{SB}$ on the mass generation of hadrons other than the pion expressed in terms of the low-lying eigenmodes. We present our results concerning $1^{--}, 1^{++}, 1 / 2^{+}$channels in the subsequent sections.

\section{$2.1 \rho\left(1^{--}\right)$}

We clearly see a bound ground state with only 32 low modes in the $1^{--}$channel in Fig. . The decay property of the corresponding correlator $\operatorname{LM}(32)$ stays almost unchanged upon including 128 low modes into the description. We observe that the mass of this bound state is found to be close to 60 percent of the original full $\rho$ meson mass. On the other hand removing 128 low modes results in a larger mass compared to both LM(128) and FULL cases. It is obvious that the masses obtained by including the low modes or excluding the low modes are not additive quantities.

We observe qualitatively the same pattern for the bound state in the $1^{++}$channel as described above under truncation level $k$. To keep everything consistent we used the same fixed set of inter-
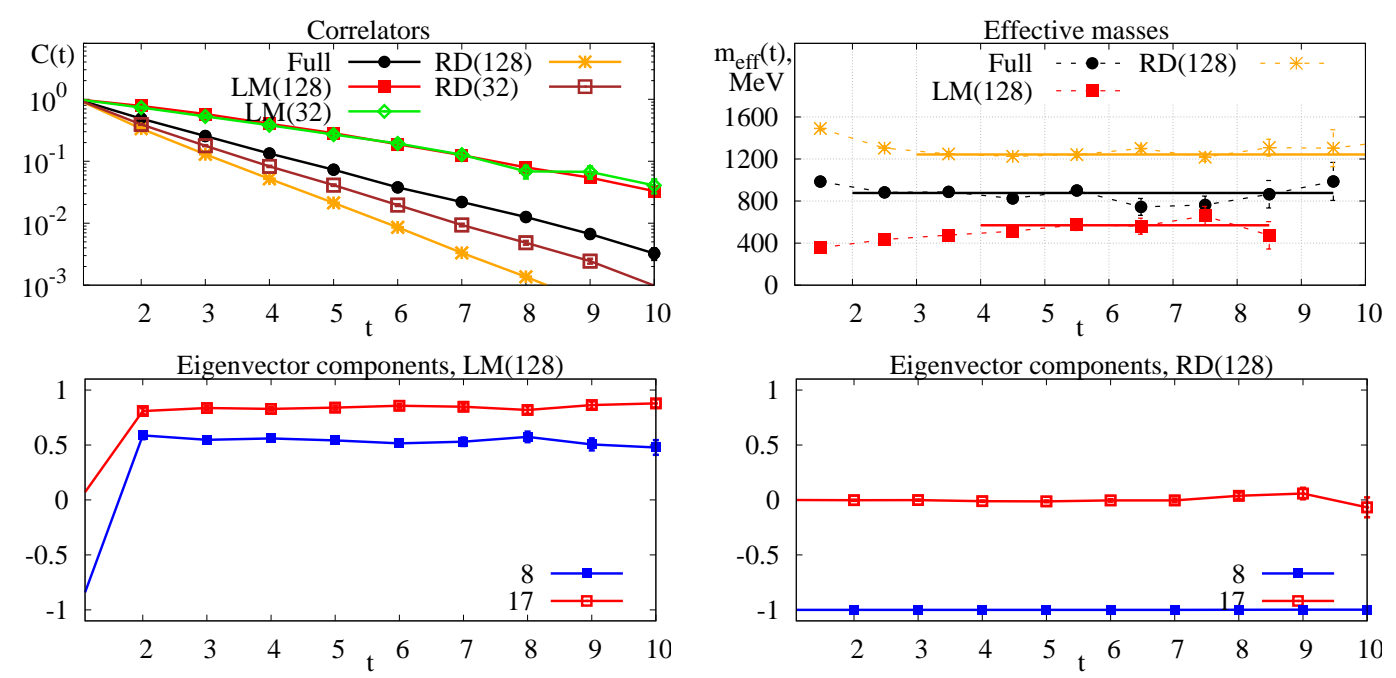

Figure 1: $\rho$ :(top left) correlators, (top right) effective masses, (bottom left/right) eigenvectors under the inclusion/removal of the low Dirac eigenmodes 

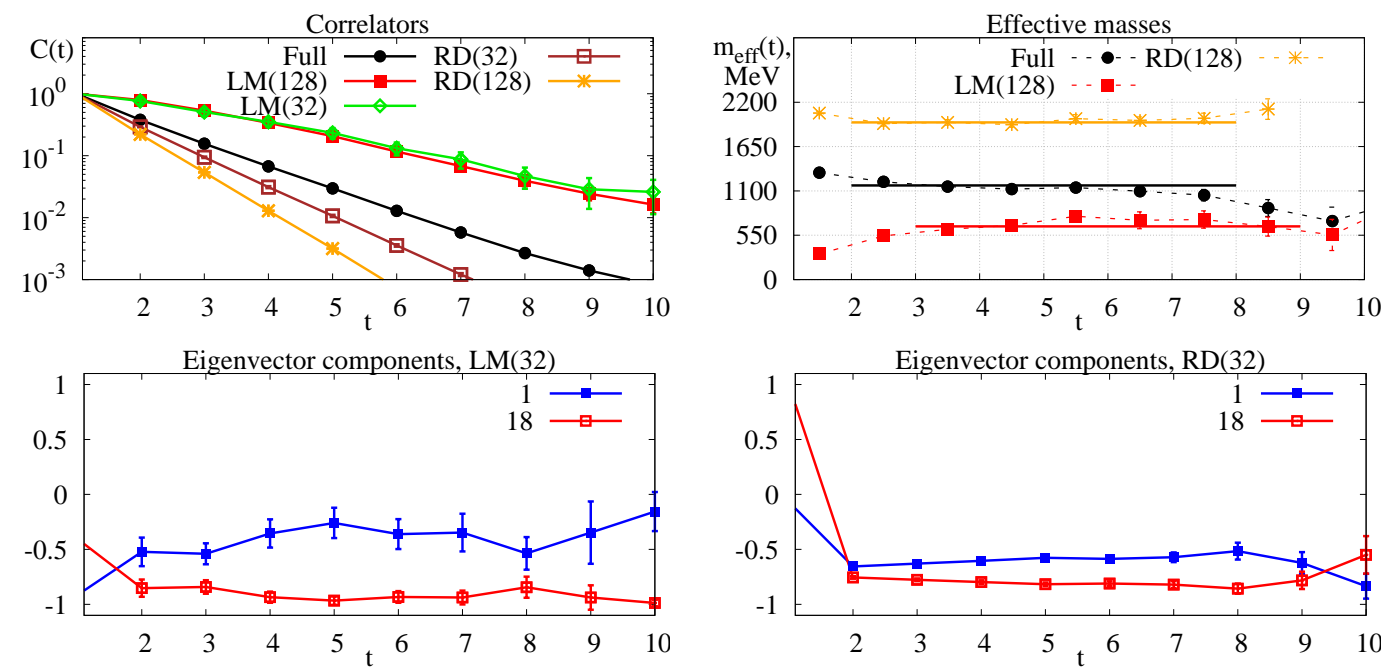

Figure 2: Nucleon: (top left) correlators, (top right) effective masses, (bottom left/right) eigenvectors under the inclusion/removal of the Dirac eigenmodes
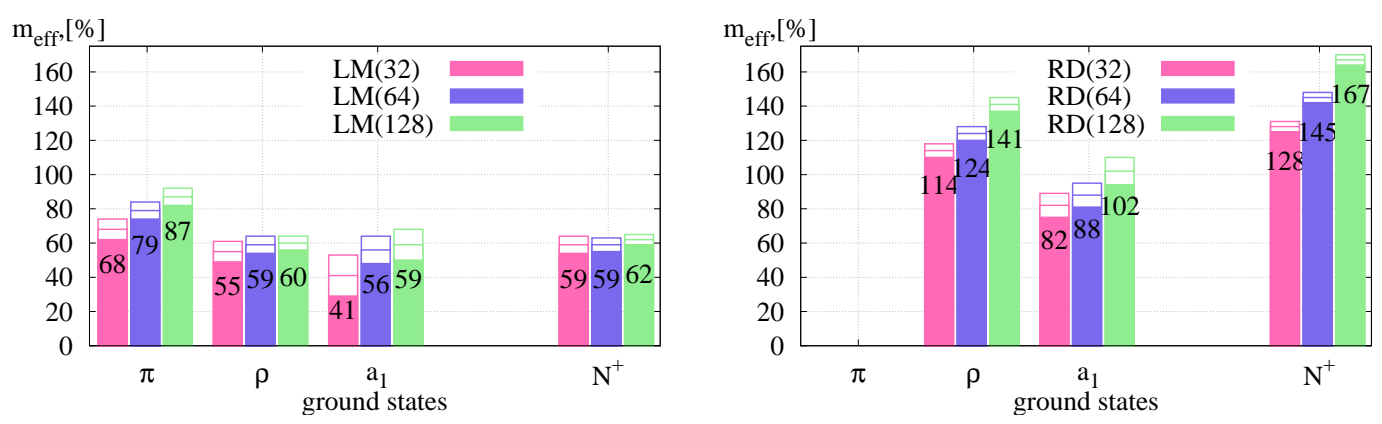

Figure 3: Evolution of the hadron masses upon the inclusion (left) and removal (right) of the low eigenmodes

polators at each truncation level, cf. Fig. W. If one optimises the rather large set of interpolators for each truncation level separately, it is possible to extend the plateau ranges.

\section{$2.2 N\left(\frac{1}{2}^{+}\right)$}

The evolution of the nucleon mass under the low mode inclusion or removal resembles the one we observed for $\rho$ meson bound state. The contribution of an increasing number of low modes starting from $k=32$ up to $k=128$ does not change significantly as it can be seen from the correlators (Fig. ㅁ). Extracting the mass of the nucleon for the case of $\operatorname{LM}(128)$ we recover almost 2/3 of the full nucleon mass. The low modes provide a large contribution to the nucleon channel of positive parity but this contribution is not enough to explain the mass of the nucleon without taking into account the effects of the high-lying eigenmodes.

\section{Conclusions}

The histograms shown in Fig. [1 illustrate the differences in the hadron mass spectrum depending on whether the low-lying modes of the Dirac operator are gradually included or excluded out of the description. 
We conclude that - unlike the mass of the pion - the masses of $\rho, a_{1}$, and $N$ grow very slowly with the number of included low modes, see Fig. [3 (left). These low modes provide a large contribution to $1^{--}, 1 / 2^{+}$channels and give a rise up to $2 / 3$ of the corresponding full masses. To fill the remaining mass for the $\rho$-meson and the nucleon one needs to take into account the high-lying eigenmodes. In contrast, it is the higher-lying modes that are the most essential for the mass of the $a_{1}$-meson. In particular, it is already sufficient to consider all eigenmodes except for the low-lying ones to obtain the full $a_{1}$ meson mass, see Fig. B] (right).

\section{Acknowledgements}

A support from the Austrian Science Fund (FWF) through the grants DK W1203-N16 and P21970-N16 is acknowledged. M.D. is supported by the Austrian Science Fund (FWF) under Grant No. DK W1203-N16. M.S. was supported by the Research Executive Agency (REA) of the European Union under Grant Agreement PITN-GA-2009-238353 (ITN STRONGnet).

\section{References}

[1] T. A. DeGrand, Eigenvalue decomposition of meson correlators, Phys. Rev. D 69 (2004) 074024, [arxiv:03]0303].

[2] G. Bali, L. Castagnini, and S. Collins, Meson and baryon masses with low mode averaging, PoS Lattice2010 (2010) 096 [arXiv:1011.1353].

[3] C. B. Lang and M. Schröck, Unbreaking chiral symmetry, Phys. Rev. D 84 (2011) 087704 , [arxiv:1107.5195].

[4] L. Y. Glozman, C. B. Lang, and M. Schröck, Symmetries of hadrons after unbreaking the chiral symmetry, Phys.Rev. D 86 (2012) 014507, [6rXiv:1205.4887].

[5] T. Banks and A. Casher, Chiral symmetry breaking in confining theories, Nucl. Phys. B 169 (1980) 103.

[6] C. Gattringer, A new approach to Ginsparg-Wilson fermions, Phys. Rev. D 63 (2001) 114501, hep-1at $/ 0003005$ ]. C. Gattringer, I. Hip, and C. B. Lang, Approximate Ginsparg-Wilson fermions: A first test, Nucl. Phys. B 597 (2001) 451, [hep-1at/0007042]. 